\title{
DIAGNOSTICS PLATE FOR SNS LINAC COMMISSIONING*
}

\author{
M.A. Plum, R.W. Garnett, R. Meyer Sr., and R.E Shafer, LANL, Los Alamos, NM 87544, USA
}

\section{Abstract}

A Diagnostics Plate is being assembled as part of the linac commissioning plan for the Oak Ridge National Laboratory Spallation Neutron Source (SNS). The D-Plate will be positioned directly after the first Drift Tube Linac (DTL) tank, where the $\mathrm{H}^{-}$beam energy will be $7.5 \mathrm{MeV}$. A full suite of beam diagnostic instrumentation is planned, including profile, current, phase, energy, and emittance measurements. The D-Plate is designed to operate up to the full duty factor of the linac, or up to $16-\mathrm{kW}$ beam power. Until the spallation neutron target is completed several years later, this will be the last time the facility can be operated at full current and duty cycle.

\section{INTRODUCTION}

A special diagnostics station, or D-plate, is being built to commission the SNS linac up through the first DTL tank. The $\mathrm{H}^{-}$beam energy at the exit of the first DTL tank is $7.5 \mathrm{MeV}$. Due to the high ( $36 \mathrm{~mA}$ after chopping) beam current and the very short range of the beam at this energy, any material that intercepts the beam is easily damaged. Only certain specially designed equipment can withstand the full $60-\mathrm{Hz}, 1 \mathrm{~ms}$ beam pulses. Interceptive beam diagnostics instrumentation must be used at reduced beam power. The D-plate is designed to perform a wide variety of measurements and functions, as summarized below:

\section{Full power $(16 \mathrm{~kW})$}

- Test and develop the front end systems (ion source, low energy beam transport, RFQ, medium energy beam transport (MEBT)).

- Measure DTL tank 1 transmission.

- Measure beam energy by time of flight (TOF).

- Full power beam stop.

Reduced power $(10 \mathrm{~Hz}, 50 \mu$ s pulse $)$

- Measure emittance with slit and collector method.

- Determine phase and amplitude set points of DTL tank 1.

- Measure wire scanner beam profiles.

- Match MEBT beam parameters to DTL acceptance. Low power

- Test permanently mounted diagnostics in the DTL beam box.

- Accommodate DTL beam aperture scans.

- Test DTL tank 1 beam steering magnets.

- Measure phosphor screen beam profile.

*Work supported by the Office of Basic Energy Science, Office of Science of the US Department of Energy, and by Oak Ridge National Laboratory.

\section{DETAILS}

Beam boxes between each of the six DTL tanks in the SNS linac contain Energy Degrader / Faraday Cup (ED/FC) devices and wire scanner actuators. The D-plate is designed to be temporarily attached to the DTL tank 1 beam box.

The D-plate will be about $3 \mathrm{~m}$ long and weigh about $400 \mathrm{~kg}$. A $300-1 / \mathrm{s}$ vacuum pump will ensure the vacuum is better than the $5 \times 10^{-6}$ Torr required to control beam loss due to gas stripping. On the D-plate there is one quadrupole magnet, one $\mathrm{x}-\mathrm{y}$ steering magnet, one current monitor (CM), three beam position monitor (BPM) pickups, an $\mathrm{x}$ and $\mathrm{y}$ slit and collector emittance measurement, a wire scanner profile measurement, a video profile measurement, an $\mathrm{ED} / \mathrm{FC}$ unit, an eightsegment halo scraper, and a full power beam stop, as shown in Fig. 1. We shall now discuss the various beam diagnostics instrumentation, working our way along the path of the beam.

The beam current will be measured with a Bergoz FCT pickup and the associated electronics now being developed for the SNS current monitor system [1]. The system is capable of measuring the current with an accuracy of $1 \%$ with a $7-\mathrm{MHz}$ bandwidth. This $\mathrm{CM}$, together with a similar unit mounted in the MEBT portion of the linac (upstream of the DTL tank), will allow the beam transmission through the DTL tank to be measured. It will also prove useful for the beam aperture scans planned for the DTL tank. Since this is a non-intercepting measurement, it can of course be used at all duty factors.

There are three BPM pickups on the D-plate. The first one has a small $2.5-\mathrm{cm}$ aperture and will be a copy of the DTL pickups that reside inside the DTL drift tubes. The second two pickups have larger, $10-\mathrm{cm}$ apertures to accommodate the increased beam size at the downstream locations. The primary function of the BPMs is to steer the beam to the beam stop. The latter two BPMs can also be used to measure the beam energy using the time of flight (TOF) method. Their $30 \mathrm{~cm}$ spacing, together with the $\pm 2^{\circ}$ (at $402.5 \mathrm{MHz}$ ) phase measurement accuracy of the BPM system [2], will allow the beam energy to be measured with an accuracy of $26 \mathrm{keV}$, which exceeds the set point accuracy specification for the DTL rf system. Using two identical BPM pickups will ensure a more accurate TOF measurement. The beam position and TOF measurements can be made at all beam duty factors since these measurements are also non-intercepting.

The first beam box on the D-plate, located $69 \mathrm{~cm}$ from the DTL end wall, contains one wire scanner mounted at 


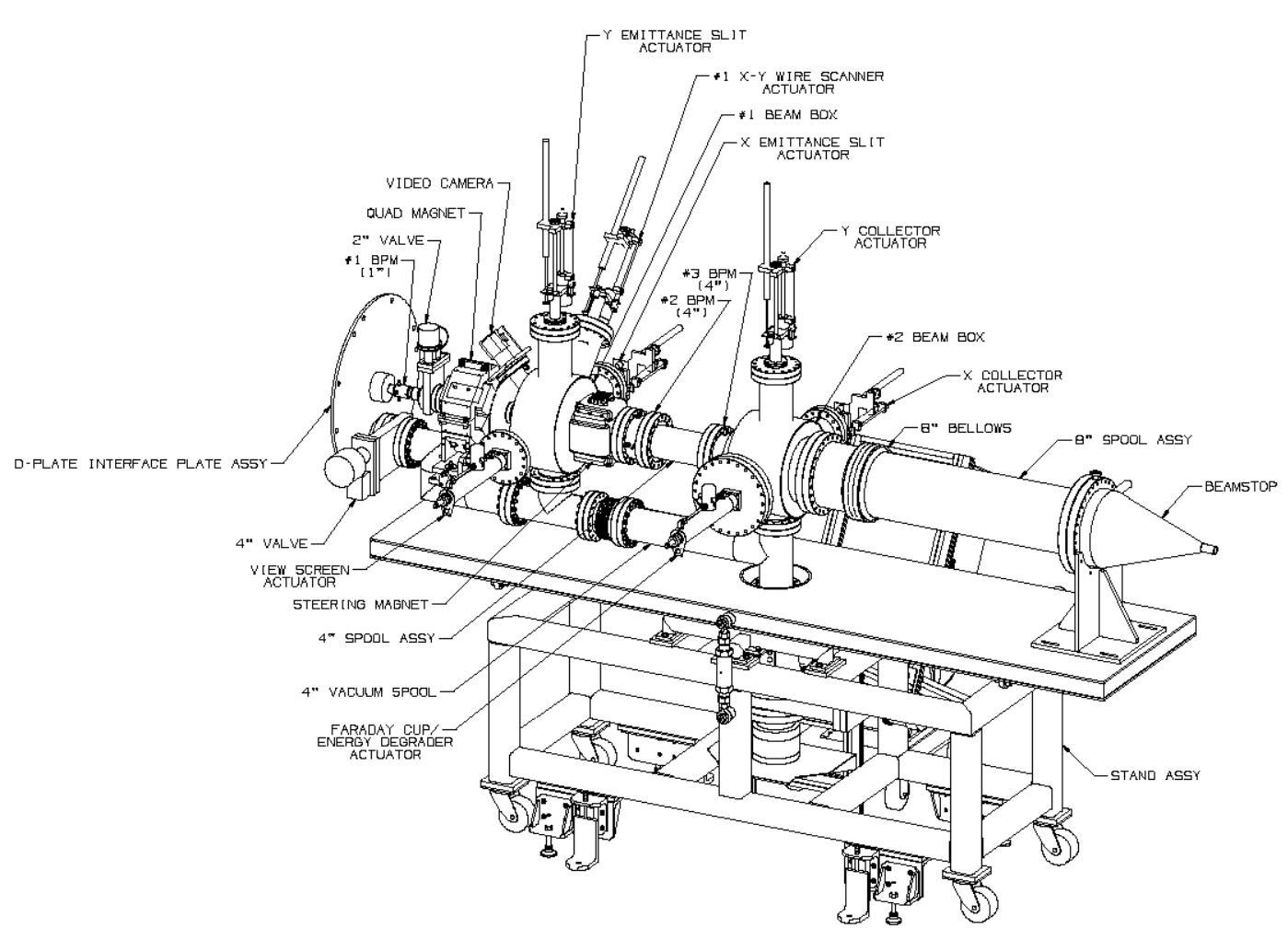

Figure 1. Line drawing of the D-plate.

$45^{\circ}$, horizontally and vertically mounted emittance slits and the phosphor viewing screen. The wire scanner will be of the same type being developed for the DTL, coupled cavity linac (CCL), and the high energy beam transport (HEBT) portions of the SNS facility. Three 30-micron diameter carbon wires mounted in the horizontal, vertical, and $45^{\circ}$ planes will measure the $\mathrm{x}, \mathrm{y}$ and diagonal beam profiles; and the beam position. Since this is an intercepting measurement, it can only be made at reduced duty factors. Thermal model calculations predict that the wires will survive for pulse lengths less than $100 \mu \mathrm{s}$ and rep rates less than $10 \mathrm{~Hz}$.

The second diagnostic in the first beam box is the phosphor viewing screen. This will allow rudimentary profile and position measurements to be made, although at much lower duty factor than the wire scanner. A video camera interfaced to frame grabber electronics and the SNS control system will be used to view and record the beam spot.

The third diagnostic in the first beam box is the $\mathrm{x}$ and $\mathrm{y}$ emittance measurement slits. Several different materials were studied before settling on graphite as the optimum material to survive the high thermal stresses required for this measurement. Even with the front faces angled at $45^{\circ}$ to spread out the energy deposition, a single $50 \mu$ s pulse raises the thermal stresses almost to the yield limit of the material. The $50-\mu$ s pulse length is necessary for the linac to ramp up to its full current and for the space charge forces to stabilize. After $100 \mathrm{~ms}$ the slit has cooled enough to receive the next pulse. The slits are therefore rated for a $50-\mu \mathrm{s}, 10-\mathrm{Hz}$ duty factor at $36 \mathrm{~mA}$. After adjusting the Dplate quadrupole magnet to optimize the tune for the emittance measurement, the beam size at the slit is about $2 \mathrm{~mm}$ rms, as shown in Fig. 2. The slit size will be a fraction of the rms size, about $0.3 \mathrm{~mm}$.

The emittance collectors are located in the second beam box, $147 \mathrm{~cm}$ from the DTL end wall. Emittance measurement electronics with 64 parallel channels are now being developed at Lawrence Berkeley Laboratory for the MEBT. We plan to use this design for the D-plate emittance measurement. With the second beam box $78 \mathrm{~cm}$ downstream of the first, 64 channels provides good angular resolution for the emittance measurement. The collector itself will be made similar to a printed circuit board, with copper signal traces etched on a ceramic substrate. The stopping length of $7.5-\mathrm{MeV} \mathrm{H}^{-}$particles in copper is $0.2 \mathrm{~mm}$, so the traces on the circuit board will be at least this thick.

Also mounted on the second beam box is the Energy Degrader / Faraday Cup (ED/FC). A 375-micron thick low-density graphite foil serves as the energy degrader. Only beam that is fully captured and accelerated in the DTL tank has the minimum $6.5 \mathrm{MeV}$ energy needed to pass through the foil and enter the Faraday Cup. A bias 


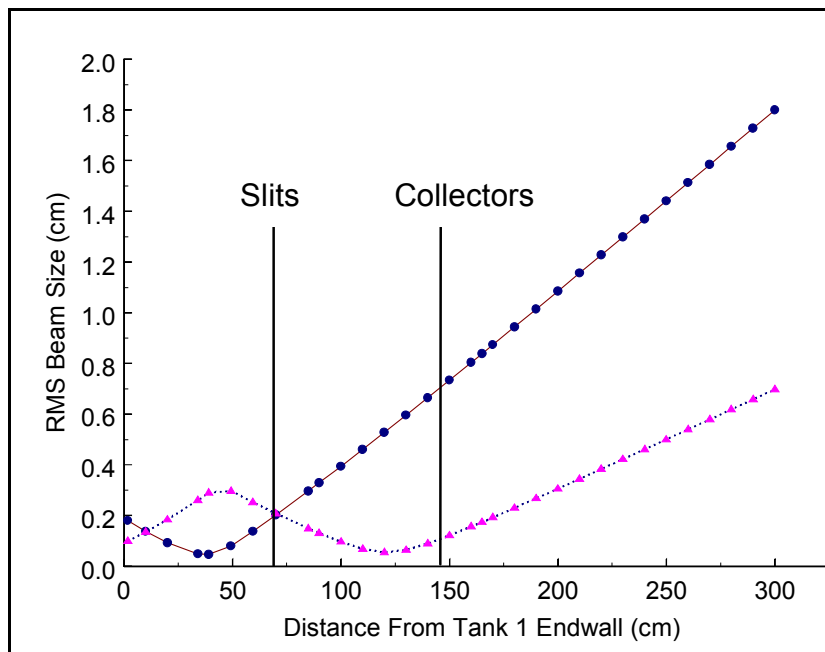

Figure 2. Plot of rms beam size vs. distance for the emittance tune. The solid line and circles show the horizontal $\mathrm{rms}$ beam size, and the dotted line and triangles show the vertical rms beam size.

ring between the ED and the FC suppresses secondary electrons to allow the FC signal to be dc coupled into an amplifier to make absolute beam current measurements. Signal strength from the FC, when plotted as a function of the DTL tank rf phase and amplitude, allows the DTL rf phase and amplitude set points to be determined. The $\mathrm{ED} / \mathrm{FC}$ on the D-plate is a higher power, larger aperture version of the unit mounted in the DTL beam box. The Dplate $\mathrm{ED} / \mathrm{FC}$ is designed for $10-\mathrm{Hz}, 50-\mu \mathrm{s}, 36-\mathrm{mA}$ (chopped) beam pulses, while the DTL ED/FC is restricted to $1-\mathrm{Hz}, 50-\mu \mathrm{s}, 36-\mathrm{mA}$ beam pulses.

The final instruments on the D-plate are the halo scraper and beam stop. The function of the eight-segment halo scraper is to measure beam halo and to assist steering the beam into the beam stop. Nickel segments $1.5-\mathrm{mm}$ thick are mounted to the entrance face of the beam stop

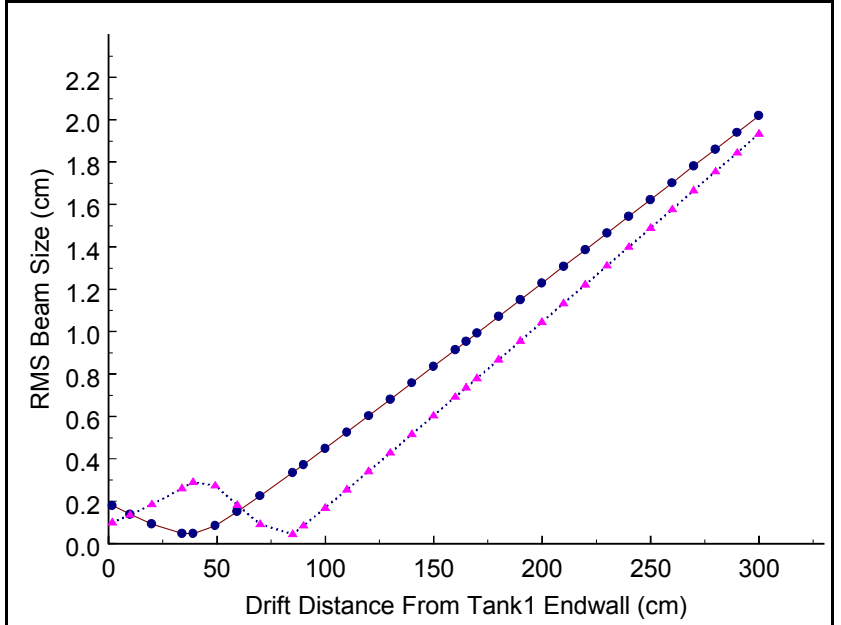

Figure 3. Plot of rms beam size vs. distance for the beam stop tune. The solid line and circles show the horizontal rms beam size, and the dotted line and triangles show the vertical rms beam size. with an aperture of $16 \mathrm{~cm}$. Kapton insulated wires attached to each segment feed the signals to a vacuum feedthrough mounted on the beam stop body. Since only a small fraction of the beam resides in the halo, this measurement can be made at full duty factor.

The beam stop [3] is designed to absorb the full $16 \mathrm{~kW}$ of beam power, corresponding to chopped (36 mA peak), $60-\mathrm{Hz}, 1-\mathrm{ms}$ beam pulses. Chopper protection circuitry installed in the MEBT will ensure that unchopped beam is never delivered to the beam stop at full duty factor. Such a situation would result in $23 \mathrm{~kW}$ of beam power, which would exceed the rating of the beam stop. The beam stop itself has a narrow conical shape with an opening halfangle of $16^{\circ}$. This design spreads out the beam deposition energy, calculated to be $277 \mathrm{~W} / \mathrm{cm}^{2}$ at the center of the beam when the D-plate quadrupole is set for high power operation, as shown in Fig. 3. The beam is stopped in a sheet of $2-\mathrm{mm}$ thick nickel, chosen to minimize the radioactivation products. Water cooling channels immediately behind the nickel conduct the heat away. Except for a booster pump to supply the high pressure water needed to cool the beam stop, the water cooling and control will be borrowed from the DTL tank 2 water skids.

\section{SUMMARY}

We have described the beam diagnostics instrumentation on the D-plate being built for the SNS linac commissioning. The non-intercepting measurements can be made at full duty factor, while the intercepting measurements must be made at a fraction of the full beam power. The D-plate will be delivered to Oak Ridge National Laboratory in the summer of 2002, and installed in the linac tunnel shortly thereafter. It will be removed from operations when DTL tank 2 is installed. At this point the linac will no longer be able to operate at full duty factor until the neutron spallation source is commissioned several years later.

\section{ACKNOWLEDGEMENTS}

We would like to acknowledge the SNS mechanical design team for their work on the diagnostics plate. Special thanks to M. Catenach, S. Ellis, D. Ireland, S. Konecni, J. O'Hara, J. Power and D. Sattler.

\section{REFERENCES}

[1] M. Kesselman, "Spallation Neutron Source Beam Current Monitor Electronics", these proceedings.

[2] J. Power et. al., "Beam Position Monitors for the SNS Linac", these proceedings.

[3] S. Konecni et. al., "Thermal and Structural Analysis of a Beam Stop", these proceedings. 